\title{
Gestión del aprendizaje y web social en la Educación Superior en línea
}

\section{Learning management and social web in Higher Education online}

\author{
Miguel Zapata-Ros \\ Universidad de Murcia. Murcia, España. \\ mzapata@um.es
}

\section{Resumen.-}

Este trabajo se puede entender como una respuesta al uso generalizado de la web social como un medio de comunicación y un marco de relación y de convivencia entre estudiantes y profesores. Como consecuencia hace énfasis en la necesidad de un marco de modelo pedagógico y de diseño instruccional que integre la web social. Plantea igualmente la necesidad de un análisis de las condiciones necesarias para la validación de ese modelo. Por último propone, a través de un análisis basado en experiencias, una serie de respuestas ante la insuficiencia de políticas instituciones que contemplen modalidades de integración y sus repercusiones.

Ofrece los resultados procesados y las conclusiones obtenidas de una investigación sobre las preferencias de los adoptadores tempranos de web social incluida en la gestión del aprendizaje por prácticas de diseño instruccional.

Con ello se ofrece a la comunidad docente universitaria propuestas, recomendaciones, modelos y el estado en cuanto a disposición docente y de desarrollo en la práctica de los profesores innovadores.

Palabras clave.- Web social, Educación Superior, Sistemas de Gestión del Aprendizaje

\begin{abstract}
.-
This work can be understood as a response to the widespread use of the social web as a means of communication and a framework of respect and coexistence between students and teachers. As a result, this text emphasizes the need for a framework of a pedagogical model and an instructional design that integrate the social web. This paper also raises the need for an analysis of the necessary conditions for the validation of that model. Finally through a system based on experience, a series of responses to the failure of political institutions that consider modalities of integration is suggested, as well as their impact.

The paper provides the processed results and the conclusions drawn from research on the preferences of the early adopters of social web included in the learning management through instructional design practices.
\end{abstract}

The final purpose is to offer the university teaching community proposals, recommendations, models and the state-of-the-art in terms of teaching and development in the practice of innovative teachers

\section{Keywords.-}

Social Web, Higher Education, Learning Management Sistems 


\section{Introducción}

Este trabajo se plantea como una respuesta ante el uso generalizado de la web social como un medio de comunicación y un marco de relación y de convivencia entre estudiantes y profesores. Haciendo énfasis en la necesidad de un marco de modelo pedagógico y de diseño instruccional que la integre. Plantea igualmente la necesidad de un análisis de las condiciones necesarias para su validación. Por último propone, a través de un análisis basado en experiencias, una serie de respuestas ante la insuficiencia de políticas instituciones que contemplen modalidades de integración y sus repercusiones.

De forma complementara, a partir de investigaciones sobre el tema, inducimos indicadores sobre calidad en entornos sociales de aprendizaje, mediante los resultados procesados y las conclusiones obtenidas de un trabajo realizado desde la Universidad de Alcalá y con el apoyo de la Red de campus Virtuales. Por último también se trata de obtener y proponer elementos metodológicos para incluir en el diseño instruccional de programas y cursos en ES (en consonancia con lo desarrollado en los trabajos y referencias teóricas de Charles Reigeluth sobre el paradigma educativo de la Sociedad postindustrial y otros trabajos en la misma línea (Reigeluth et al, 2008; Reigeluth, 2012; Zapata-Ros, 2014)).

Otro objetivo no menos importante es ofrecer a la comunidad docente universitaria, y a los gestores, propuestas, recomendaciones, modelos y el estado en cuanto a disposición docente y de desarrollo en la práctica de los profesores innovadores.

Por lo demás el trabajo se inserta en un proceso de investigación más amplio, dentro de un proceso en la línea de diseño instruccional con investigación formativa.

Pero hagamos un poco de historia. Podemos constatar que a principios de la década (Zapata-Ros, 2011a) los campus virtuales se habían convertido en una realidad que ocupaba un espacio de acceso, habitual y mayoritario. Así lo aseguraban los informes más conocidos en el ámbito global, en EE.UU. y en España también: El informe Going the Distance: Online Education in the United States, 2011, de Sloan (Allen \& Seaman, 2011), y en España el informe UNIVERSITIC2011, publicado por el CRUE (Uceda et al, 2011). Sin embargo en estos informes se hacía énfasis en aspectos exclusivamente tecnológicos. No se abordaban cuestiones de metodología docente, aprendizajes o evaluación, ni de otras variables de diseño educativo. Tampoco abordan la aparición de entornos de web social ni su integración en entornos y con fines educativos. Se estaba perdiendo, según analizábamos, con ello una oportunidad de innovación metodológica. Coincidía entonces la ausencia de experiencia de uso de web social, y de planteamientos de integración en los entornos virtuales, con el hecho que entonces constatábamos y que hoy persiste: Los LMS se han transformado de forma generalizada en entornos de gestión de la actividad convencional que se realiza alrededor de la educación -de la gestión de las calificaciones, de fichas de alumnos, de tiempos, actividades y recursospero en muy pocos casos gestionan el aprendizaje que se produce en el sentido que señalamos en el trabajo "Analítica de aprendizaje y personalización" publicado en la revista de la Red de Campus Virtuales (Zapata-Ros, 2013a):

"La web social confiere a los sistemas de gestión del aprendizaje de una potencia anteriormente desconocida. Ya se está implementando casi de forma generalizada el uso de la web social de propósito general (Facebook, Google+, Twitter, con herramientas como Drive, Hangouts, o con modalidades audiovisuales como Youtube) o específica, como sucede con Mahara y Moodle, como complemento vinculado a la instrucción o simplemente de forma complementaria e incluso en algunos casos 
espontánea. Esta potencia permite construir este espacio que es la base de la personalización, pero que en sí no la es. De hecho en una primera fase los profesores pueden realizar recogidas y análisis de datos que suministren elementos para la evaluación formativa y para la evaluación del progreso en el aprendizaje, elementos de identificación etc., directamente entrando en el espacio de los alumnos y analizando los elementos que constituyen esa información. En un trabajo más elaborado y sistematizado (como hemos visto que se hacía con ELLIS) se pueden obtener perfiles completos de aprendizaje de los estudiantes. En definitiva, y en todo caso, los profesores, e investigadores, tienen a su disposición, pueden acceder, a una amplia gama de recursos en forma de enlaces a páginas web, artículos, referencias de libros, etc. solo que ahora esto significa el acceso forma continua con las referencias dentro del campo y de unas referencias a unos intereses y a unas características personales del alumno. Y sobre todo pueden acceder a trabajos en proceso o desechados, a un material gris que normalmente suministra más información que el trabajo ya concluido, sobre la metodología de trabajo del alumno, caminos emprendidos y desechados, intuiciones, etc."

Sin embargo las cosas cambiaro en el panorama global. En el informe Horizon de 2015 (Johnson et al, 2015), que como sabemos indica las predicciones en tres plazos, a corto (1 $\circ 2$ años), medio ( $3 \circ 4$ años ) y largo (5 ○ 6) para su adopción, en el apartado a corto plazo señalaba un incremento en el uso del blended Learning (Increasing Use of Blended Learning) y dice (Pag 16):

"los instructores de programas eficaces de aprendizaje mezclado (b-learning) deben encontrar formas de estimular las actividades sociales y el pensamiento crítico dentro de un entorno en línea - tal y como se espera de ellos en las experiencias directas"

Consecuentemente con ello extraen Implicaciones para la política, el liderazgo, o la práctica:
"Muchas instituciones de educación superior reconocen la necesidad de directrices concretas de aprendizaje en línea y están tomando la iniciativa para idear políticas eficaces. La Universidad de Glasgow, por ejemplo, publicó "E- Learning Estrategia 2013-2020", un documento técnico que describe las mejores prácticas para el campus y aumenta el alcance y la accesibilidad de los métodos de aprendizaje en línea que el profesorado puede adoptar. Entre sus prioridades está la uso de un entorno virtual flexible que incorpore características interactivas para hacer el aprendizaje más social para los estudiantes. En lo que respecta a los programas de aprendizaje en línea también la Universidad Estatal de California se plantea cómo se puede mejor gestionados y organizados a nivel institucional y departamental."

En todo caso son iniciativas pioneras, como después veremos, que indican una tendencia.

El mismo informe en el apartado "Blending Formal and Informal Learning. Solvable Challenge: Those that we understand and know how to solve" señala la dificultad de lucha contra las inercias existentes: 
"Los enfoques tradicionales de enseñanza y aprendizaje con raíces en el siglo 18 $y$ anteriores son muy comunes en muchas instituciones, $y$ a menudo sofocan todas las innovaciones en el aprendizaje que los pueden inhibir."

Viendo en la mezcla del aprendizaje formal, lo que se enseña en esas instituciones, con el aprendizaje informal, lo que se aprende en la calle, en Iternet o en las relaciones interpersonales, un peligro:

En la medida que (Johnson et al, 2015) Internet ha traído consigo la capacidad de aprender algo acerca de casi todo de forma inmediata (sin mediación), existe un interés creciente por las modalidades de aprendizaje basado en la curiosidad, autodirigido, que tiene un origen común en los museos, en los centros de ciencia, y en las redes de aprendizaje personal.

En distintas partes el informe señala las iniciativas que manifiestan la tendencia. Así en este mismo apartado (Johnson et al, 2015), en la pág. 22, señala, entre muchas otras referencias, informes como el "Learning in a Digital Age" que estudia el creciente uso de blogs, wikis, podcasts, redes sociales y otras herramientas como vehículos para profundizar el learning.

Muy importante es lo que señala (Johnson et al, 2015) en la página 35, donde hace una clasificación en tecnologías con un capítulo de Social media technologies

\begin{abstract}
Algunas universidades están quedando por delante de la curva y han desarrollado sus propias plataformas de aprendizaje adaptativo. Este es especialmente el caso en el sector de la educación con fines de lucro; en 2013, se concedió una patente a la Universidad de Phoenix para su plataforma de aprendizaje adaptativo "“Academic Activity Stream" " - una inversión de mil millones de dólares. "Academic Activity Stream" es similar en apariencia y funcionalidad a las redes sociales, la clasificación de información para los estudiantes basados en sus intereses únicos, historial de rendimiento y objetivos de aprendizaje. Del mismo modo, la Universidad de Michigan creó "Gradecraft", una plataforma en línea que fomenta la toma de riesgos y múltiples vías hacia el dominio que los estudiantes avanzan en curso material. El entorno "Gradecraft" se gamifica, permitiendo a los estudiantes para ver cómo influyen en sus decisiones directamente cómo así que absorben y demuestran su comprensión del nuevo material a medida que avanzan de nivel ${ }^{1}$.
\end{abstract}

\footnotetext{
${ }^{1}$ Some universities are staying ahead of the curve and have developed their own adaptive learning platforms. This is especially the case in the for-profit education sector; in 2013, a patent was issued to the University of Phoenix for its adaptive learning platform "Academic Activity Stream" - a billion dollar investment. "Academic Activity Stream" is similar in appearance and functionality to social networks, ranking information for students based on their unique interests, performance history, and learning objectives. Similarly, the University of Michigan created "Gradecraft," an online platform that encourages risk-taking and multiple pathways towards mastery as students progress through course material.264 The "Gradecraft" environment is gamified, enabling students to see how their choices directly impact how well they absorb and demonstrate their understanding of new material as they move from level to level.265
} 
Hasta muy tarde, desde las istancias oficiales, como en nuestro caso es la CRUE no se hacen eco de la importancia ni tan siquiera de la existencia de la web social como ámbito educativo. El informe "UNIVERSITIC 2013: Situación actual de las TIC en las universidades españolas" (Durán et al, 2013) no hace mención alguna a los entornos y herramientas sociales dentro del ámbito de la gestión del aprendizaje, no aparece ninguna mención a red social, web social, Facebook, blog, wiki, Mendeley, Research Gate, Academia.edu, o a cualquier otro término relacionado, ni en los bloques temáticos dedicados a gestión de la investigación, ni a la docencia, y solo aparece una vez la expression "redes sociales" pero vinculada a la gestión de la ciencia. Es en la en la pág. 92, en el EJE 7 DE GESTIÓN: PRINCIPALES PERSPECTIVAS TIC, en la Tabla 2.10: Indicadores de Gestión del Eje 7: Principales expectativas y tendencias TIC (descripción), dentro del Objetivo 7.1. Identificar las principales tendencias y expectativas TIC del equipo de gobierno, en el apartado Liderazgo y Gobierno $\mathrm{Tl}$ aparece como descriptor el Conocimiento en Abierto, Comunicación Social, movilidad y colaboración con las siguientes características:

Edición y producción digital; publicación de la productividad científica, de materiales docentes y de documentación corporativa. Licencias y propiedad intelectual. Redes sociales. Necesidades tecnológicas para la difusión y divulgación de la Ciencia. Políticas de movilidad. Iniciativas BYOD (Bring your Own Device). Alianzas.

Evidentemente se trata de una orientación con la mención de un área a desarrollar en la gestión de la ciencia y de la investigación que tiene en cuenta a las redes sociales como elemento de comunicación social de la ciencia. De forma tangencial observamos, no sin curiosidad, que una tendencia propia del elearning, y bien caracterizada por la literatura específica, como es el BYOD (Bring your Own Device) aparezca en la gestión de la ciencia.

En cualquier caso como vemos, en contraposición a lo que sucede en el Informe Horizon, se trata de una referencia muy parcial y limitada.

Por otra parte se constata en el marco internacional de vanguardia de la Educación Superior, en el Informe Franklin (Armstrong and Franklin, September 2008) la asunción y el uso generalizado por los alumnos dela web social, particularmente las redes sociales. Estos entornos constituyen "habitats" donde los profesores innovadores, los que adoptan de forma temprana las metodologías y entornos innovadores, los "early adopters $^{2}$ ", están intentando nuevas cosas de forma esporádica y aislada sin el apoyo institucional. Así en nuestro país la Web social se utiliza en casi todos los ámbitos de la educación superior, incluyendo las áreas académicas y de apoyo. Estas áreas tienden a

\footnotetext{
2 "Earlier adopters in a system also differ from later adopters in personality variables. Earlier adopters have greater empathy, less dogmatism, a greater ability to deal with abstractions, greater rationality, greater intelligence, a more favorable attitude toward change, a greater ability to cope with uncertainty and risk, a more favorable attitude toward science, less fatalism and greater self-efficacy, and higher aspirations for formal education, higher-status occupations, and so on" (Rogers, 2010).
} 
constituirse en "puntos calientes" donde los "early adopters" están ensayando sus innovaciones, muy lejos de ser todavía prácticas generalizadas o incluidas en el marco formal institucional, o incluso con herramientas de diseño instruccional aún entendidas de forma individual en asignaturas, o de grupo en áreas o departamentos. Tiene sentido pues recoger y estudiar con relación a la Educación Superior (ES) de nuestro país, donde parece ser que, en cierta medida, la infraestructura técnica está disponible para que los estudiantes y los profesores puedan acceder a las funciones de web social, los requisitos y rasgos que ha de tener la innovación para que esta se haga de la forma más eficiente. Se trata pues de cuestiones de calidad.

Hasta la fecha el uso de la web social ha sido impulsado principalmente por los intereses particulares de los miembros individuales del personal en lugar de las políticas institucionales. Los profesores están utilizando la Web 2.0 para mejorar su enseñanza a causa de las posibilidades que ofrece, o porque los estudiantes están utilizando ya las tecnologías, con el compromiso, o porque son tecnologías que los estudiantes van a utilizar después de la graduación en la vida profesional. Por último hay razones de calidad en el aprendizaje: Con nuevas formas, la web social favorece la colaboración y la interacción reflexiva y autónoma de los estudiantes, así como la evaluación de su trabajo por el profesor en el mismo punto donde se produce el proceso de elaboración o de realización de la tarea.

No es tampoco de descartar el análisis y la reflexión que desde la perspectiva de las comunidades de aprendizaje se hace de los LMS combinados con los entornos sociales como hábitats de comunicación y de aprendizaje compartido (Vázquez-Martínez y Cabero-Almenara, 2015).

Más recientemente se ha hablado (Zapata-Ros, 2018) de un nuevo tipo de aprendizaje que necesita desarrollar nuevas pedagogías y pedagogías inteligentes, como son microaprendizajes, aprendizajes oportunistas o de las nuevas formas de evaluar y de diseñar la instrucción asociadas.

Por todo ello es el momento pues de realizar una revisión metodológica, aceptando el hecho de que los entornos virtuales de aprendizaje, merced a la potencialidades pedagógicas que encierran, se han constituido, a pesar de ser fenómenos aislados o esporádicos, en nuevos entornos sociales de aprendizaje en la ES. Este fenómeno, por el mismo imperativo que lo hacían los anteriores sistemas de aprendizaje, no puede escapar de la reflexión sobre la calidad. Los docentes, gestores, alumnos e investigadores necesitan referencias claras y comunes sobre criterios de calidad basados en consensos. En este caso también.

En el presente trabajo hacemos una reseña de experiencias relevantes sobre este tema, incluyendo una reseña sobre una investigación propia sobre la percepción que tienen los "early adopters" en nuestro ámbito (educación superior en España) y extraemos una serie de conclusiones. 


\section{Casos y experiencias. Necesidad de elementos metodológicos para una docencia que integre la web social en los sistemas de gestión del aprendizaje}

Es aceptado comúnmente que la universidad se ve y se verá concernida por la web social como sucede con el resto de instituciones, servicios o negocios. Pero además en este caso sus funciones y actividades específicas, la gestión de la educación, del aprendizaje, la enseñanza y la Evaluación se verán especial y fuertemente afectadas. Además de la otra misión de la Universidad, la investigación, como se señala en "La ciencia compartida" (Zapata-Ros, 2011b). Pero al contrario de como sucede en otros sectores no servirá solo como instrumento de contacto y de relación de los integrantes de las comunidades universitarias, o como ampliación de la participación de otras comunidades que interactúan con las distintas actividades que la universidad tiene encomendada, incluso después de la carrera como instrumento de comunicación entre comunidades profesionales o de exalumnos. Sería un error considerarlo sólo así. Habrá cambios de calado como se dice en el trabajo citado y en "“Evaluación de la calidad en entornos sociales de aprendizaje" (Zapata-Ros, 2011a). Ni la web social es el único conductor de estos cambios, en realidad la Web social es sólo una parte del ecosistema de la Educación Superior (ES). Pero otros elementos son de mayor importancia: Los cambios metodológicos que induce ella en connivencia con otros aspectos, la personalización, la analítica, etc. influyen y presionan para una mayor eficiencia en el aprendizaje y en la docencia. Igualmente los cambios de comportamiento de la población estudiantil en estos entornos lo hace así, lo posibilita más. Tiene repercusiones en la mejora del aprendizaje y de los métodos de enseñanza.

Pero además la web social es, en particular, una tecnología con un profundo potencial de cambio que induce en el sector de la ES. Sobre todo en lo que tiene que ver en la forma de aprender y de enseñar, cambios que deben repercutir en el diseño educativo. Estamos hablando de que los ámbitos de aprender que se producen, al ser abiertos y por los efectos catalíticos de las tecnologías de la web social, particularmente atractivos para los alumnos, es lo que permite una mayor independencia y autonomía de los estudiantes, una colaboración más fluida, y por ende se debe producir un aumento de la eficiencia pedagógica.

En la revisión de la literatura, y en el estado del arte sobre experiencias, que hemos realizado hemos considerado dos grupos. Uno el de los precedentes (casos) de experiencias relevantes que han sido comunicadas en artículos y contribuciones reseñadas en Google Académico hasta 2015, buscadas con palabras relevantes, o con fragmentos textuales relevantes. Y otro ha sido el de precedentes de elaboración de aportaciones teóricas, constructos e ideas que en conjunto puedan contribuir a dar un marco teórico y una pedagogía sobre la integración de entornos sociales como ámbito de enseñanza y de aprendizaje universitario. En este caso hemos seguido la misma metodología que en el primero. 


\subsection{Precedentes de experiencias o de modelos de práctica docente}

Son escasos. Reseñamos lo más significativo y relevante, que incluso lo podemos señalar como referentes. Son ejemplos seleccionados por Alice Marwick (September, 2008) en el informe más utilizado sobre el tema con respecto a EE.UU.

1.

El informe UKOLN (Web 2.0 in Higher Education in the United Kingdom: Observations on the growth of Web 2.0 and social Web technologies from a JISC-funded national adviser ) fue financiado por el Consejo de Museos, Bibliotecas y Archivos, el Joint Information Systems Committee (JISC). Su autor es Brian Kelly (2008) de UKOLN y University of Bath. Forma parte como otros relativos al estado de la web social en la educación superior en otros países (EE UU, Holanda, Sudáfrica y Australia) de un informe más amplio titulado $A$ review of current and developing international practice in the use of social networking (Web 2.0) in higher education.

Está orientado a documentar las experiencias y reflexiones personales del autor sobre el crecimiento del uso de la Web 2.0 en el sector de educación superior del Reino Unido. Es un informe descriptivo sobre el uso de las tecnologías Web sociales dentro de la comunidad de educación superior del Reino Unido. Como en nuestro caso el informe intenta proporcionar información estratégica. Solo que en este caso intenta describir la justificación para el uso de los distintos tipos de tecnologías web sociales en todos los ámbitos universitarios, no exclusivamente en cuanto a diseño instruccional, calidad o elaboración de nuevos modelos de intervención docente, sino reflexionar sobre las implicaciones organizativas de un mayor despliegue de estas tecnologías. El informe ofrece un análisis sobre la utilización de tecnologías de la Web social, proporcionando para ello pruebas documentadas de la retroalimentación que se ha obtenido en el uso de las tecnologías web sociales y por el examen de los comentarios y discusiones de quienes participan activamente en el suministro y el uso de dichas tecnologías.

En general señala sólo casos institucionales sin entrar, salvo excepciones que después veremos, en casos concretos de aplicación a las actividades propiamente educativas. Señalan casos institucionales, presencia en las redes como $\mathrm{Fb}$, consideraciones sobre el papel y la actitud de alumnos y profesores hacia el medio etc. Igualmente hace consideraciones sobre desarrollos de redes sociales Elgg y Ning, pero sin señalar modelos de integración en el diseño de cursos o de carreras ni de la generación de metodologías docentes que las incluyan.

En 2004 y 2005 solo refleja como actividad conferencias donde se habla del potencial de la web social (con referencias explícitas a blogs, wikis y servicios de mensajería instantánea). Esto sucede a pesar de los eventos nacionales de alto perfil que se organizaron en 2004 , en 2005 . Se constata que en la práctica hay "actividades poco importantes" y que "tal vez esto era un reflejo de la lenta asimilación de lo que puede 
ocurrir después de una ráfaga inicial de la actividad por los primeros adoptantes, ya que se caracterizan por la híper-curva de Garner".

Únicamente se reseña una excepción, en la charla sobre "Using Networked Technologies To Support Conferences" dada en la conferencia EUNIS 2005. En ella se describe el potencial de las tecnologías de la Web colaborativa para su uso en conferencias y otros eventos celebrados en lugares que proporcionan acceso WiFi. En el citado documento se describen varios experimentos tempranos en uso de las tecnologías para fomentar discusiones y debates en línea, en los eventos, así como se razona el potencial de tecnologías como Skype para que los participantes remotos a participar. Como vemos está muy lejano del uso educativo de redes sociales, y mucho más de su inclusión en actividades programadas.

Este hecho da idea del nivel de desarrollo del uso instruccional de la web social.

La irrupción de la web social en la universidad se produce en el Reino Unido en 2006. Como medio de comunicación institucional fundamentalmente y como medio de comunicación social entre los alumnos.

Pero solo se dan charlas en las que se ponen de manifiesto su beneficios potenciales. Fueron "el inicio de una serie de charlas en varios eventos en todo el país que pusieron de relieve los beneficios potenciales de lo que ahora se estaba denominado Web 2.0". “(...)la gestión de Internet y las comunidades de desarrollo Web dentro de las instituciones universitarias del Reino Unido eran muy conscientes de que la Web 2.0 podría tener un papel jugar para apoyar las actividades de enseñanza y aprendizaje, la investigación y el apoyo de las instituciones. Las instituciones que ahora tenían que hacer frente a los problemas para identificar y superar los obstáculos con el fin de integrar servicios Web 2.0 en las principales actividades institucionales".

Sin embargo no tiene un reflejo en la literatura en los años siguientes en los que los informes insisten en el hecho de que en todas las conferencias y congresos se insiste en los beneficios potenciales, hasta hoy día, sin ir acompañados de una práctica confirmada, evaluada y validada.

En el informe se señalan los casos de los primeros adoptantes de esta tecnología como medio de comunicación institucional y en todo caso, aceptando la masiva incorporación de alumnos usuarios, que establecen políticas de dotación de infraestructura de redes sociales estándares o especificas (Elgg y Ning). Coincide con lo reseñado en el informe.

2.

El informe Web 2.0 in Higher Education in the United States of America de Alice Marwick (September, 2008), Universidad de Nueva York, Forma parte como los anteriores del Informe Franklin (Armstrong and Franklin, September 2008). Tiene gran importancia porque los Estados Unidos es un líder mundial en el uso de la tecnología Web 2.0. Gran parte de la investigación sobre el uso de los medios sociales, y las experiencias más 
significativas, se llevan a cabo allí. Todas estas tecnologías se inventaron en Estados Unidos, y los estudiantes estadounidenses son muy versados y precursores en el uso de medios sociales. Como resultado hay numerosos ejemplos de medios sociales utilizados para fines educativos en las universidades. Muchos de ellos además son impulsados por las teorías pedagógicas más recientes, por modelos de aprendizaje activo, centrados en el estudiante y constructivistas que se corresponden de forma ajustada a las affordances de la web social.

Según el informe en EE UU se da la tensión más fuerte entre las experiencias más avanzadas, las iniciativas de web social impulsadas desde las propias instituciones, que en muchos casos son los agentes más dinamizadores, o la corriente de abajo hacia arriba de los innovadores tempranos, por un lado y la resistencia más fuerte y la desconfianza por parte de los sectores más tradicionales y conservadores del profesorado.

A nivel federal hay políticas gubernamentales, como las disposiciones anti-intercambio de archivos de la versión actual de la Ley de Educación Superior, que potencialmente afectarán a las opciones para implementar tecnologías de medios sociales.

Por otro lado la mayoría de los departamentos de ayuda en TI y de las universidades en su conjunto aún no integran la Web 2.0 en los campus virtuales porque por lo general están comprometidos con los sistemas LMS heredados, y con frecuencia son resistentes al cambio por autoridades y profesores. Contrasta con que las universidades han adoptado políticas tecnológicas institucionales que incluyen medios de comunicación social. Se trata sin duda de un recurso de imagen y marketing.

Presenta casos como el de la Universidad Xavier donde los servicios de la universidad a los estudiantes de correo y de red social ante la falta de eficacia han sido sustituidos por los portales, páginas web y blogs de estudiantes en redes convencionales integrados en los servicios de la universidad. Y también como el impulso del movimiento Open Access, ha afectado por su propia naturaleza el que las universidades acepten los entornos 2.0 creados alrededor de esta alternativa. Así señala el caso de la Facultad de Artes y Ciencias de la Universidad de Harvard que aprobó una resolución que exige publicar trabajos académicos en línea. Esto supone la eliminación de requisitos y cuotas de suscripción para ver los trabajos académicos. De esta forma (Alexander, 2008) los contenidos educativos publicados de esta forma quedan igualmente abiertos, lo que contribuye a un campo de conocimiento público. Dicho contenido puede estar recogido y utilizado en guías, revistas de educación, y artículos de opinión sobre metodologías docentes. Y aunque las revistas de Open Access no son medios de comunicación social per se, las normativas universitarias como la de Harvard de contribuir a una mayor apertura sobre la producción de conocimiento, la mayor parte de las veces contribuyen al uso de la web social que se crea en torno a estas actividades.

Alice Marwick (September 2008) en su interesante informe toma de Tim O’Reilly (2006) una conceptualización de la interactividad vinculada a la web social. 
Tim O'Reilly ofrece un continuo del carácter de interactividad a lo largo de una clasificación que hace de las aplicaciones Web 2.0 (o más bien de los usos de las aplicaciones Web 2.0): En un extremo están los usos que no dependen de la conectividad a Internet, y que podría existir muy bien sin el Internet (por ejemplo, la versión electrónica de un diccionario Español-Inglés). En el otro extremo estarían las aplicaciones que no podrían existir sin Internet y la conectividad de red (como por ejemplo Facebook). Esto no es caprichoso pues la interactividad sería dependiente en mayor o menor medida de la realidad o de la red. Hablaríamos de un mayor o menor grado de la componente "web social" de la interactividad.

Y con arreglo a este criterio clasifica los ejemplos de académicos que utilizan la tecnologías Web 2.0

En un extremo estarían los que imitan las aplicaciones sociales del mundo real, pero no aprovechar el verdadero poder de la interactividad supuestamente intrínseca a los medios sociales. Así esta el ejemplo de Sarah Hulburt, profesora de Whitman College. Para ella y sus alumnos los blogs de clase existen solamente para los estudiantes que presentan trabajos. Ella lo justifica: "Mientras que las estructuras tradicionales de asignación permiten hacer la transferencia a la estructuras del blog con bastante claridad, de manera que no sea una transformación automática de lo real en un trabajo interactivos como resultado, sino como una continuación (...). El blog si se considera como trabajo a realizar puede muy fácilmente convertirse en no más que una forma complicada de presentar un trabajo individual ". Si un profesor desea cosechar los beneficios de la interactividad, debe diseñar tareas "reales" que se beneficien de las características interactivas de la Web 2.0.

De esta forma Marwick (September 2008) continúa describiendo lo que sucede en EE UU: En términos generales, el uso académico de las tecnologías Web 2.0 se puede dividir en dos tipos: Una la de los profesores que utilizan la Web 2.0 para crear o difundir material de clase, y otra en la que sean los estudiantes los creadores de los contenidos, ya sea de forma independiente, con un instructor, o como resultado de una tarea de clase. Mientras que el primero es probablemente el uso más frecuente en los Estados Unidos que el segundo, el que utiliza el verdadero potencial de la Web 2.0 es el que se encuentra en las clases y las tareas, y que se nutre de los talentos de los estudiantes para inspirar la colaboración y la creación de contenidos.

A continuación Marwick (September 2008) señala que como sucede en el resto de casos tratados hay excelentes ejemplos de "prácticas educativas con visión de futuro, ya que se prestan muy bien a la integración de la teoría y la praxis" académica

Describe tres ejemplos significativos de diferentes asignaturas que utilizan una variedad de aplicaciones web 2.0 para promover los objetivos de la clase. Se trata indudablemente de ejemplos donde de forma explícita o implícita hay un diseño instruccional claro. Intentaremos glosar los casos señalando estos aspectos.

Primer ejemplo: BJ Fogg, de Stanford, con la creación de aplicaciones de Facebook. 
El Dr. Fogg vinculó una unidad didáctica que impartió (taught a class) con un grupo Facebook y con otros dispositivos sociales (blogs y wikis). Correspondía a una experiencia del departamento de Ciencias de la Computación (The Stanford Persuasive Technology Lab, 2007). Ya el vincular la unidad didáctica con el entramado de dispositivos implica elementos de diseño no fáciles de resolver: Actividades, evaluación, formas de relacionarse entre los alumnos y el profesor, etc. Pero el diseño era más elaborado: Además del grupo había aplicaciones Facebook, pero básicamente la unidad contemplaba un grupo privado y un grupo público, un blog, una wiki y una extensa página web (con tecnología blogger facilitada por Silicon Valley empresarios y desarrolladores de software) donde los alumnos publicaban sus proyectos y recibían comentarios.

Esta estructura de clase (class, como se le llamó) fue un éxito. Dos versiones del modelo que se generó a partir del proyecto tuvieron más de 100.000 (1 millón) de instalaciones. BJ Fogg escribió: "Como docente e investigador, me parece que tratar de frente con Facebook es el enfoque correcto. Los estudiantes son impactados con proyectos relacionados con Facebook. Responden bien a las tareas que les doy a través de un canal de Facebook". Este sin duda era el factor visible del éxito y de atractivo en la comunicación, pero el factor clave estaba en rodearlo de un diseño adecuado de actividades y de comunicación, en el proceso de realización de la tarea, entre alumnos.

3.

El informe Web 2.0 for Content for Learning and Teaching in Higher Education (Franklin and van Harmelen , 200728 May) contiene una guía y repertorio de interesantes prácticas de cuatro universidades inglesas. Se extraen una serie de interesantes conclusiones sobre esta práctica de las cuales recogemos las más importantes que se identifican con lo visto en nuestro trabajo. Esta información es recogida y ampliada en el informe UKOLN (Web 2.0 in Higher Education in the United Kingdom...) de Brian Kelly, University of Bath.

En el informe principal, ofrece un análisis de la Web social, junto con una recopilación de los sistemas más frecuentemente utilizados para la educación. A continuación examinan el caso de cuatro universidades inglesas que han adoptado un enfoque estratégico e implementado servicios de web social de manera diferente al nivel institucional.

En todos los caso ponen al servicio de los estudiantes herramientas de web social. En algunos recomiendan y se da un uso educativo a los recursos posibilitando iniciativas puntuales y favoreciendo un habitat para los alumnos en los que pueden crear entornos personales. Pero en ningún caso, incluso en el de la Universidad de Edimburgo donde instan a iniciativas de uso regular ("predicar con el ejemplo") no hay elementos de diseño instruccional emanados desde la propia institución. 
En el informe se sigue por consideraciones acerca del contenido de la Web social, su creación y uso, junto con una identificación de las cuestiones que afectan la creación de contenido y uso. Es en la siguiente sección donde se consideran los impactos de la web social en el aprendizaje, la evaluación, la docencia y temas importantes relacionados con la pedagogía y la evaluación. Se describen por un lado las aportaciones más relevantes sobre las nuevas características de los estudiantes, sus perfiles de competencias que han sido modificados por la práctica cotidiana y temprana de útiles y herramientas tecnológicas. Fomentando ciertas capacidades de relación y de comunicación y disminuyendo otras de elaboración, de reflexión, o en general de todas aquellas en las que la respuesta del proceso de elaboración se produzca en el individuo y no en la máquina. Pero en todo caso constata que se está en presencia de un aprendiz distinto de cuyos cambios tiene que hacerse eco la organización pedagógica de la universidad en todos sus niveles.

También resume las aportaciones de la teoría del aprendizaje clásica que ya se han abordado en muy diversos trabajos, estudios e informes (socioconstructivismo, etc).

Sin embargo contrasta esta necesidad por tener en cuenta el nuevo escenario con las conclusiones del informe en este campo:

1. Mientras que algunos ejemplos de enfoques pedagógicos específicos, mencionados más arriba, sí funcionan, la consulta reveló una fuerte sensación de que los educadores aún no saben cómo incorporar el uso de la web social, si bien existe un convencimiento de que la tecnología sí interrelaciona con el Aprendizaje y la Enseñanza para su mejora y en la demanda de unas nueva pedagogía y unos nuevos métodos de evaluación.

2. La naturaleza cambiante de los estudiantes que ingresan ahora a la Educación Superior, requiere respuestas, posiblemente en direcciones diferentes. Por un lado un lado suministrando formas nuevas de acoplamiento de los alumnos matriculados, dando opciones a formas distintas de ambientes en los que se tenga en cuenta su capacidad para relacionarse, comunicarse y trabajar utilizando los nuevos ambientes tecnológicos. Y por otro se plantea la necesidad de una formación correctiva. De manera que en los niveles anteriores, por ejemplo en la educación primaria y Secundaria se haga énfasis en una formación reflexiva. (Pág. 21)

Concluye el informe con la política institucional y la estrategia a considerar para un uso en la ES en que se obtengan mayores beneficios para sus fines y un mayor impacto social de éstos.

Señala que "debido a la relativa inmadurez de la tecnología y la experimentación con su uso, es demasiado pronto para formular recomendaciones específicas en la mayoría de las áreas". Y que "lo más importante es que, debido a que el uso de la Web social en diversas áreas de aplicación (aprendizaje, enseñanza, administración, gestión) se encuentra todavía en una etapa temprana, se recomienda que las instituciones adopten 
regulaciones no estrictas, en la experimentación con las tecnologías que ayudan la implementación de medidas pedagogías".

Hasta ahora la situación no ha pasado como en el resto delos casos vistos de reconocer la importancia de estas tecnologías emergentes y favorecer los servicios a alumnos y a profesores, así la Universidad de Gales, Newport, tomó la decisión en base a una conferencia de "apoyar las tecnologías existente (o emergentes) de la Web 2.0, tales como blogs, wikis, etc.", y de "convertirse en un, servicio ágil de adaptación [se supone que de dichos servicios], y de apoyar rápidamente esas nuevas tecnologías." Desde entonces ha desplegado un servicio de blogs para sus estudiantes $y$, más recientemente, ha establecido un entorno de red social para la comunidad estudiantil. No habla nada de otros apoyos o experiencias.

La Universidad de Warwick hizo algo parecido y solo ofrece, ella y el informe, datos numéricos sobre uso sin profundizar o analizar en qué consiste el uso. Que se supone es de exclusiva comunicación. Habla exclusivamente de blogs: "El servicio de blogs Warwick sugiere que la Universidad logró su objetivo la creación de comunidades con 5.235 blogs, 10,8461 puestos y 192.195 comentarios"

Un caso más productivo es el de la La Universidad de Edimburgo. La clave fue una conferencia sobre el tema " Social technologies: from pioneers to mainstream use?" que se celebró en la Universidad de Edimburgo en noviembre de 2006. Con mucha y selecta participación se estudiaron las innovaciones en proyectos Web 2.0 que se están produciendo dentro de la Universidad y de cómo los estudiantes estaban haciendo uso de las tecnologías de la Web Social. Así como de los diversos problemas, entre ellos los riesgos legales y de los peligros de depender de los servicios de terceros (entiéndase Facebook).

Como aportaciones relacionadas con la docencia y con el diseño instruccional presentaron las siguientes conclusiones:

- Las tecnologías sociales están haciendo a los docentes repensar su papel.

- El aprendizaje en línea es cada vez más participativo que receptivo, más basado en contenidos colaborativos. Las tecnologías móviles son cada vez más importantes.

- Los estudiantes usan herramientas de redes sociales todo el tiempo, compartiendo información que de otra forma no compartirían. Lo cual les pareció sorprendente.

- No todos los estudiantes usan el software de colaboración social.

- Si se consideran los aspectos legales en el inicio de cualquier proyecto, la ley se puede considerar una ayuda. La ley está ahí para facilitar, no para penalizar. Creative Commons ofrece un marco legal y políticas de uso aceptables si se usan adecuadamente.

- Los riesgos de no hacer nada son mayores que el riesgo de hacer algo: "Las universidades suelen tardar mucho tiempo para adoptar nuevas tecnologías. La inercia 
institucional puede ser un obstáculo importante para la adopción de las tecnologías de la web social".

- Ciertas preocupaciones institucionales como algunas ideas sobre el control de la calidad, los contenidos adecuados, la privacidad de datos, la intromisión de la administración, etc. están en contra del espíritu de la Web 2.0. La web social, en una primera fase debe ser refractaria a los filtros. Debe ser receptiva y favorecer la alimentación de grandes cantidades de datos generados por los usuarios.

- Esto planteaba la que después ha sido idea central: ¿Sigue siendo pertinente, eficiente y rentable, para la Universidad la prestación de servicios (por ejemplo el correo electrónico, la agenda) utilizando el método tradicional de la selección, el apoyo, el mantenimiento y los servicios centralizados?

- Para los estudiantes, las herramientas comerciales a veces son mejores que las herramientas provistas por la Universidad (por ejemplo, el sistema de correo electrónico de la universidad les da muy poco espacio de almacenamiento, se cae, no funciona durante periodos, no se coordina con otros servicios (lo de cursiva lo añadimos nosotros) y sobre todo los alumnos no lo pueden mantener en su vidad profesional una vez que han dejado la universidad). La alternativa (asegurando el papel de acreditación que hoy ofrece el correo corporativo de la Universidad, y Google ofrece esa posibilidad) es asociar la prestación.

En definitiva señalaban la necesidad de un cambio en la cultura. Las políticas de uso deben ser revisadas. Se necesitan modelos más sofisticados de lo que las normas encorsetan, que permitan la accesibilidad abierta.

Otra cuestión importante que señalaba el informe es el ecosistema heterogéneo que supone la universidad (sobre todo las grandes como la de Edimburgo) donde las innovaciones se dan de forma espontánea y muchas veces no solo desconocida por la institución sino por sus colegas cercanos. Esto nos priva de auténticas buenas prácticas de uso de la web social incluso con un diseño instruccional que puede ser explícito pero no formal.

Hay bolsas de innovación, e incluso se reproduce en unidades más pequeñas, con bolsillas de innovaciones. La Universidad tiene que desentrañar esas experiencias, junto con las desviaciones o malos usos. Hay que alentar a los innovadores para compartir y aprender, incluyendo sus errores.

Y concluyen diciendo que un punto que merece ser destacado es que:

Los riesgos de no hacer nada son mayores que el riesgo de hacer algo. Las universidades suelen tardar mucho tiempo para adoptar nuevas tecnologías. La inercia institucional puede ser un obstáculo importante para la adopción de las tecnologías web social. 
Además de los señalados sobre el Reino Unido y EE.UU., en el informe Franklin (Armstrong and Franklin, September 2008) podemos encontrar otros tantos informes parciales de Holanda, Sudáfrica y Australia que no ofrecen sustanciales aportaciones sobre experiencias a lo visto en los informes anteriores, aunque sí ofrece un informe de síntesis cuyas aportaciones más interesantes ofrecemos en la segunda parte de este punto y en el resto de este trabajo.

\subsection{Precedentes de elaboración de un marco teórico.-}

Como sucede con otros entornos y herramientas tecnológicos, la creciente popularidad y difusión de las redes ha inducido a pensar, de forma simple, que al proporcionar las redes nuevas vías y propósitos para la comunicación entre estudiantes y profesores, las comunicaciones sociales pueden convertirse en un factor de éxito del aprendizaje. En este contexto la interacción ha sido reconocida como un indicador clave de la calidad de los cursos en línea. Lo cual en un análisis más elaborado con experimentos, análisis empíricos e investigaciones puede efectivamente proporcionarnos la evidencia de que ciertos usos de las redes debidamente utilizadas en el contexto de un diseño minucioso pueden favorecer determinados aprendizajes por medio de ciertos elementos de interacción en las actividades.

Estas experiencias y su ulterior análisis nos han proporcionado ya algunos elementos de elaboración para un marco teórico que a continuación glosamos

3.1

Roblyer y Wiencke (2003), en el desarrollo de una matriz de valoración para evaluar la interacción en los cursos en línea, identificaron cinco componentes: el diseño para la interacción social, el diseño para la interacción instruccional, las affordances que favorecen la interactividad, la participación de los estudiantes y el compromiso del profesor. De manera que en un todo sistémico cada tipo de interacción contribuye a la calidad global y el potencial impacto de un curso en línea.

\section{2}

Schwartz (2009) también considera que "la teoría de la tutorización" ("mentoring theory") puede proporcionar una base teórica para la definición de un papel de instrucción para las redes sociales. Schwartz cita a Ragins \& Kram (2007) en su 'trabajo sobre "tutoría relacional" en The Handbook of Mentoring at Work: Theory, Research and Practice, cuando dicen que

"... existe un potencial de desarrollo, incluso en una sola interacción (por ejemplo)" (p. B13). El modelo de tutoría relacional sugiere que "... el crecimiento en las relaciones ocurren cuando dos personas experimentan... aumento de la energía y el bienestar, la posibilidad de tomar medidas, el aumento de conocimiento de sí mismo y de otros, un impulso a la autoestima, y un interés en más de conexión" (pág. B13). 
Como profesor de educación superior, Schwartz (2009) juzga sus propias experiencias con los estudiantes en Facebook como el cumplimiento de la mayoría, si no todos, estos criterios y por tener beneficios comparables a una reunión después de la clase. Por lo tanto, el intercambio que se produce en las redes sociales, teóricamente, puede ser a la vez una forma válida de comunicación de instrucción altamente interactivo y una oportunidad para el asesoramiento pedagógico.

\section{3}

Anderson, T. (2006) en Teaching a distance education course using educational social software concluye que el Software Social Educativo encontrará su nicho real a su propio ritmo, con la autointegración continua de profesores y alumnos, el aprendizaje informal, permanente y otras formas de aprendizaje autodirigido.

Al contrario de lo que sucede con otras herramientas y otros entornos (léase realidad aumentada, mundos virtuales, e incluso pizarra digital) donde hay más literatura y expectativas que experiencias prácticas ( y las pocas que hay estarán muy publicitadas) en el caso de experiencias que integren software social, o más específicamente redes sociales, son muy pocas las que están muy reseñadas, y menos aun las que repercutan en un cambio sustancial en el diseño instruccional que estén reseñadas y descritas y hayan generado publicaciones. Sin embargo hemos detectado que, al menos en un sector bien definido existe una relativa abundancia de experiencias de uso de software social integrado en la práctica educativa con un cierto nivel de programación educativa.

4.

Alice Marwick (September 2008), en Web 2.0 in Higher Education in the United States of America, hace otra aportación a las ideas para una pedagogía de la web social, se trata de una construcción de abajo hacia arriba: Los nuevos hilos conductores de esta pedagogía proceden de profesores innovadores, comunidades de docentes y de los propios estudiantes. El fundamento pedagógico para el uso de la Web 2.0 en el aula tiene que ver con los cambios tanto en los estudiantes (los creadores de contenidos, los entusiastas de los medios digitales) y los cambios en las teorías del aprendizaje. Se plantea el aprendizaje conceptualizado como un proceso de colaboración participativa de abajo hacia arriba. De igual forma a como se plantea en las teorías del aprendizaje centrado en el estudiante, el constructivismo y el aprendizaje activo. Y de la misma forma la pedagogía de la Web 2.0 coincide con esas teorías en los principios fundamentales sobre las ideas de comunidad, creatividad, participación y reflexividad.

Con relación a la cultura participativa y a la producción de conocimiento, Henry Jenkins (2009), profesor del MIT sostiene que la Web 2.0 está facilitando un nuevo tipo de producción de los medios de comunicación colectiva de abajo hacia arriba, que él llama "cultura participativa", facilitado por "la explosión de las nuevas tecnologías de los medios que hacen posible que los consumidores utilicen los nuevos medios para 
archivar, anotar, apropiarse y recircular el contenido multimedia de nuevas y poderosas formas ". Jenkins sostiene que a medida que los jóvenes, que antes eran únicamente los consumidores de medios, se están convirtiendo productores de medios aparecen nuevos tipos de habilidades y fortalezas para la pedagogía, pero también nuevos problemas. Entre estos destaca:

- La brecha de participación: el acceso desigual a las oportunidades, experiencias, habilidades y conocimientos necesarios para participar plenamente en el mundo que se está construyendo.

- El problema de la transparencia: Los desafíos a los que se enfrentan los jóvenes para aprender a ver claramente las nuevas formas de comunicación.

- El desafío de la ética: Las formas tradicionales de relación profesional tienen que adaptarse a la socialización del conocimiento, y esto acarreará problemas de plagio, propiedad intelectual, autoría, etc.

La teoría de la cultura participativa, de Jenkins hace hincapié en que los estudiantes "han dejado de ser consumidores pasivos y se han convertido en productores activos de conocimiento." Por lo tanto, los modelos de arriba hacia abajo de aprendizaje caracterizado por formas más antiguas de la pedagogía deben ser modificadas para incorporar y fomentar este tipo de la actividad creativa en colaboración, que ya es valioso en sí mismo. La creación de nuevos medios de comunicación a partir de piezas de otros productos de medios permite a los estudiantes trazar conexiones y relaciones entre los tipos aparentemente dispares de información.

Este punto de vista no sólo aboga por que los estudiantes se animen a utilizar las nuevas herramientas de comunicación para crear contenido, resolver colectivamente los problemas y hacer "mash-up" o "remix" de distintas obras, sino también para que los problemas sociales generados por estas prácticas se aborden directamente en la educación.

\section{Conclusiones de los precedentes.-}

En lo analizado se constatan tres conclusiones que podría generalizarse a cualquier innovación con tecnología en la educación universitaria.

La conclusión general primera es que la mayoría de los usos educativos de la Web 2.0 se hacen sobre una base $a d-h o c$, por el profesorado motivado, el personal y los estudiantes afectados. Y estas experiencias la mayor parte de los casos no son escalables. 
La segunda conclusión general es que, para realmente aprovechar los comportamientos colaborativos e interactivos facilitados por las tecnologías de medios sociales, previstos y posibles, la coordinación de esfuerzos se debe hacer a nivel de toda la universidad.

Y por último la conclusión general tercera es que las experiencias de uso de web social integrada en un diseño instruccional explícito son escasísimas (solo hemos podido detectar tres o cuatro). Pero las que hay, aún con solo algunos elementos de diseño instruccional explícito o con implicaciones que hacen percibir un fuerte diseño implícito, son muy valiosas y fructificas.

Además podemos ofrecer una serie de conclusiones obtenidas directamente de los precedentes analizados.

Existen una serie de problemas que son comunes en todos los países con una infrestructura común en las universidades que ofrece a los estudiantes una tecnología estándar de web social. El primero es la sensación generalizada común a las Instituciones de educación superior y a sus estudiantes, de que se encuentran ante unos territorios inexplorados que constituyen los usos educativos de las tecnologías Web 2.0.

Existe igualmente la conciencia de que los límites en los que la información y las comunicaciones, que hasta ahora han sido exclusiva de las universidades y controlados por ellas, están desapareciendo. Están perdiendo ese control. Y las universidades más conscientes están luchando por recuperarlo, o al menos para dar sentido a una forma de operar en ese espacio modificado y permeable.

Hay un nuevo estado de responsabilidad en los estudiantes. Tienen que descubrir las consecuencias de sus declaraciones públicas en la red, en la evaluación, en la percepción de sus competencias y actitudes, y en la proyección sobre su futuro profesional. Y esto debe ser asumido por las instituciones.

Los ideas, la composición de lugar y los marcos de referencia que hemos utilizado hasta ahora ya no son adecuados. Muchas fronteras se han vuelto difusas, entre lo físico y lo virtual, entre la vida personal, académica, profesional y social, entre el aprendizaje formal e informal, entre el consumo y la producción de conocimientos.

El uso de la web social para la enseñanza y el aprendizaje y para el apoyo al estudiante es considerable (Armstrong and Franklin, September 2008), aunque desigual, dentro de las instituciones de educación superior en los países desarrollados. Algunos de los desarrollos actuales están impulsados institucionalmente o al menos son compatibles con el curriculum, pero la gran parte de lo que se hace está siendo realizado por el personal de forma voluntarista, con un interés o entusiasmo exclusivamente personal. Es poco o nulo lo que se ha hecho en las políticas nacionales para integrar la web social de forma seria en el contexto de las actividades o de la evaluación, incluso con orientaciones y atención a los informes. Sin embargo, sí hay constancia de ejemplos del uso de la Web 2.0 para apoyar a políticas puntuales o ya existentes, tales como el uso 
de redes sociales para apoyar a los estudiantes antes de llegar a la universidad como parte de la agenda de integración.

Uno de los problemas que habrá que afrontar es el de la identidad. Los propios estudiantes y las instituciones tendrán que enfrentarse a asegurar la identidad y la naturaleza de los estudiantes y en general del personal en línea.

Se tenga o no respuesta para la integración de la web social en el marco de la educación, la falta de nuevos modelos pedagógicos creará incertidumbre para el personal académico y de gestión y entre los estudiantes.

Otra preocupación constatada la produce un considerado inevitable cambio de cultura en los académicos: La rápida expansión y enorme de información accesible a través de la web, pero sobre todo unida a las herramientas que se pueden utilizar para reutilizar $y$ crear nuevos conocimientos en línea han creado un entorno muy diferente de trabajo y de comunicación.

También existe la constancia de que estos problemas son comunes para los estudiantes y en mayor o menor grado (en la medida que son conscientes) para las autoridades y para los profesores, en todos los países en los que están comprometidos con el uso de herramientas de la web social.

\section{También hay conclusiones respecto de las ventajas:}

Se acepta comúnmente que la web social ofrece un conjunto de affordances que no se encuentran en otras tecnologías. Sobre todo en lo que respecta a la co-creación de conocimiento y el apoyo a las actividades de colaboración. Incluso de forma trasversal a la estructura de las universidades y de los países.

Los estudiantes ya están, ya llegan a la universidad, utilizando estas tecnologías, por lo que están comprometidos con ellos. Se encuentran dispuestos a utilizarlas en su aprendizaje.

También se acepta que la web social incluye la capacidad de agregar información, datos e ideas procedentes de diferentes lugares de forma rápida y sencilla y que ese material sigue estando a disposición del estudiante una vez que han dejado la universidad.

La influencia de la universidad en los espacios externos es mayor y más fácil, sobre todo si te utilizan en el aprendizaje y la enseñanza.

\section{Conclusiones sobre directrices.-}

En cada uno de los diferentes casos, situaciones y países estudiados existen en mayor o menor medidas directrices sobre integración de la web social en los estudios, sobre todo en los servicios, pero son siempre limitadas y parciales. El informe Franklin (Armstrong and Franklin, September 2008) dice que "son muy pocos los [casos de países] en los que se han creado estrategias formales para la integración y el uso eficaz de las tecnologías 
Web 2.0 en todas las instituciones". En su informe no presentan ninguno, y no hemos podido encontrar no solo casos de países sino de instituciones con estrategias formales de integración conducentes a un diseño integrado.

Destaca la frecuencia de directrices que denotan una preocupación o ansiedad ante malos usos o problemas derivados del uso por estudiantes de redes sociales. Así se han desarrollado códigos de conducta en torno al uso, tratando de dar sentido y justificación a las cuestiones éticas y de protegerse a sí mismos, y al personal y a los estudiantes a través de las regulaciones explícitas. Las instituciones parecen estar muy precavidas por los desarrollos Web 2.0 y observan los hábitos de los jóvenes con cuidado para tratar de entender la forma en que deberían responder.

Como caso frecuente podemos señalar directrices sobre limitaciones de espacio físico (memoria), de la necesidad de apoyo y el deseo generalizado de ir por delante de los acontecimientos (manifiesto en expresiones como "La generación estudiantil cambiante" y también "la universidad tiene que seguir el ritmo de los acontecimientos.")

A continuación señalamos directrices recogidas a nivel de profesores o de gestores de servicios que puedan tener sentido en un diseño a este nivel. Ni que decir tiene que esto se produce como consecuencia de que la mayor parte del desarrollo que aparece dentro de las instituciones de educación superior (IES) es impulsado a partir del trabajo de las personas que son innovadores o que son los primeros en adoptar y reconocer el potencial de la funcionalidad ofrecida por las herramientas Web 2.0, como sucede en el estudio que nos ocupa:

- Tener en cuenta que la continuidad de los recursos (creados por los estudiantes y otros) va más allá de la vida universitaria ("Quiero que mis estudiantes tengan acceso a los recursos después de que termine la asignatura o los estudios")

- Propiciar la creación de comunidades que vayan más allá de las comunidades tradicionales universitarias: empresarios, profesionales, otros estudiantes en otros países. ("Propiciar el compromiso con una comunidad más amplia. Trascendencia de las fronteras institucionales o físicas")

- Propiciar el desarrollo de las comunidades antes y después de los estudios de grado y de postgrado: Crear, en redes sociales, sitios y grupos de ex alumnos

- Propiciar el compromiso con el aprendizaje permanente. Sobre todo a partir de la formación y del ejemplo en valores dado por los docentes como valores de los académicos: "Los alumnos tienen que confiar en el uso de la web social en su futuro laboral (y también pueden beneficiarse de una perspectiva de aprendizaje permanente)."

- Utilizar la web social para la extensión de nuevas formas de conocimiento. (Manifestar como valor el estar "Interesado en las formas en que las nuevas tecnologías permiten que se produzcan nuevas formas de conocimiento, publicó, y en que se evalúan".) 
Muchos de estas directrices, sobre todo las que se enuncian como valores, son impulsadas por las redes profesionales docentes.

También hay conclusiones sobre directrices para el curriculum universitario: Es frecuente percibir la necesidad de formación para una alfabetización en la web social, algunos consideran (Armstrong and Franklin, September 2008) esto debe constituir una parte formal del plan de estudios. Lo consideran necesario en los estudios de de comunicación e informática. O bien para la inserción en un futuro laboral, la práctica profesional, el aprendizaje permanente y la vida social.

\section{Mejora de los métodos de enseñanza tal como lo ven los profesores}

En general se puede decir que hay un número considerable de profesores innovadores que, como sucede en otras profesiones, buscan actualizar sus métodos y poner en práctica nuevas ideas, en este caso sobre el uso de herramientas Web 2.0. Esto es visto simplemente como parte de su vida profesional.

De esta forma hay, como sucede en otras corporaciones en las universidades debe existir una Unidad Profesional para mejorar las prácticas de enseñanza (y mantenerlas al día) y esta unidad debe contemplar el uso de herramientas que contemplan la colaboración, el seguimiento y el proceso (Seguimiento de los progresos), el uso de herramientas de comunicación distintas de las herramientas convencionales "cerradas", y los posibles cambios en las prácticas de evaluación.

Por otro lado la integración de la web 2.0 es compatible y es explicada sobre todo por los enfoques pedagógicos sustentados por las teorías constructivistas y social constructivistas: Están más comprometidas con la idea de que el conocimiento es una construcción social y las tecnologías de la Web 2.0 abundan en esa idea de forma visible y tangible. $Y$ también con el aprendizaje colaborativo: Hay nuevas oportunidades para la creación y la co-creación de contenidos. Para crear colaborativamente los recursos de aprendizaje.

\section{Estudiantes y aprendizaje.}

Otras conclusiones que se obtienen lo son con respecto a la mejora del aprendizaje de los estudiantes. En muchos casos, hay una visión basada en las experiencias de estudiante $\mathrm{y}$ en la percepción de un deseo de utilizar estas tecnologías porque entienden que existe un provecho "con la Web 2.0 para mejorar sus oportunidades de aprendizaje a través de la colaboración, las diferentes herramientas de comunicación y por un mayor acceso a los recursos, propios y ajenos". Se basa en el principio de que "los jóvenes ya se han apropiado de estas plataformas."

\section{Incertidumbre Pedagógica}

Sin embargo se detecta igualmente un sentimiento de incertidumbre entre algunos profesores y autoridades, por dos razones 
- No están seguros acerca de la utilidad pedagógica de las herramientas Web 2.0. (Piensan que "no tiene un uso práctico para mejorar las cosas, y es sólo la tecnología por sí misma", "sería una inversión de educación en tecnologías no probadas ".)

- Hay una falta de confianza acerca de disponer las pedagogías adecuadas para hacer un uso eficiente de estas tecnologías.

\section{Identidad y personalidad.-}

Un problema inevitable a abordar es la identificación de la personalidad delos estudiantes en la web social. Y otra cuestión a tener en cuenta es que en los próximos años vamos a ver cómo los estudiantes adquieren una personalidad múltiple, y tienen algún grado de separación entre su personalidad de trabajo/estudio personal y sus personalidades sociales.

\section{Sobre desarrollos futuros en los que se utilice la web social.-}

En lo que hemos visto hay algunos desarrollos futuros que ya podemos identificar y serán importante para tener en cuenta cuando se integren de forma institucional o en el diseño instruccional de profesores, grupos o departamentos. Se pueden prever varias vertientes:

\section{Diseño instruccional}

- Nuevas oportunidades curriculares utilizando el acceso a informaciones de tipo primario como son fuentes de datos y elaboraciones y medios de comunicación a través de los canales de la Web social para actividades y procesos colaborativos.

- Nuevas oportunidades de evaluación. Hay procesos que hacen posible rastreary registrar a través de las aplicaciones Web social la producción de los alumnos y el desarrollo de las tareas. Los grandes datos permitirán obtener perfiles de aprendizaje y personalizar la educación.

- El desarrollo e integración de nuevos entornos de aprendizaje virtuales (incluidos los entornos personales de aprendizaje) que se basan en las tecnologías de la web social.

\section{Desarrollo profesional y propedéutico.-}

- El carácter y las affordances de las tecnologías sociales se usarán para proporcionar soporte común a los alumnos antes de que lleguen a la universidad.

- La web social permitirán rentabilizar conocimientos, métodos de trabajo y relaciones adquiridas en la universidad en la vida profesional.

Difuminación de fronteras y disminución de la capacidad de control de las universidades 
- A largo plazo se debe producir una difuminación de los límites de las instituciones, que se volverán más permeables, con los entornos virtuales de aprendizaje, a lo de fuera de la institución. Dando cabida incluso a las personas que no son miembros de la institución, y a conocimientos que residen fuera de la institución.

- Se producirá una difuminación entre el aprendizaje formal y el informal.

- Se producirá una reducción en la capacidad de las instituciones para el control de la tecnología que los estudiantes utilizan en su aprendizaje. Y

- Una reducción en la capacidad de las instituciones para controlar el acceso a la información que los estudiantes utilizan en su estudio.

\section{Gestión}

- Se extenderá el uso de sistemas de gestión de identidad para proporcionar acceso a un número cada vez mayor de alumnos de los dos sistemas en que se basará el aprendizaje el externo y el de la universidad.

\section{Conclusiones prácticas para los sistemas tecnológicos.-}

- Es una constante la idea central siguiente: ¿Sigue siendo pertinente, eficiente y rentable, para la Universidad la prestación de servicios (por ejemplo el correo electrónico en el caso más claro pero también en otras aplicaciones como la agenda o las listas de distribución) utilizando el método tradicional de la selección de herramientas, el apoyo, el mantenimiento y los servicios centralizados?

- Se ve en todos los casos que, para los estudiantes, las herramientas comerciales muy frecuentemente son mejores que las herramientas provistas por la Universidad (por ejemplo, el sistema de correo electrónico de la universidad les da muy poco espacio de almacenamiento, se cae, no funciona durante periodos, no se coordina con otros servicios y sobre todo, los alumnos, no lo pueden mantener en su vida profesional una vez que han dejado la universidad). La alternativa (asegurando el papel de acreditación que hoy ofrece el correo corporativo de la Universidad, y Google ofrece esa posibilidad) es asociar la prestación con un correo estándar (gmail, Microsoft, etc.).

- El uso de sistemas no estándares, exclusivamente universitarios, puede significar que los estudiantes tienen que hacer uso de muchos más nombres de usuario y contraseñas, y que su espacio de aprendizaje se fragmenta.

- Las aplicaciones de la web social reemplazarán cada vez más las aplicaciones de escritorio (en particular el uso de herramientas de productividad gratuitas como Google Docs en lugar de Microsoft Office).

\section{Conclusiones sobre Teorías del Aprendizaje}


Se ha hablado de la Teoria de la Cultura Participativa de Jenkins (2009), pero también habría que hablar de la revisión de las teorías del aprendizaje existentes como son las del:

\section{Aprendizaje Activo}

La teoría del aprendizaje activo enfatiza el aprendizaje descentralizado - la colaboración, la interacción en red-, en lugar de la difusión del conocimiento de arriba hacia abajo. Se centra en el estudiante, que controla su propio proceso de aprendizaje. El aprendizaje activo es el adecuado para destacar un problema del mundo real, en cuya solución incluye la observación de un fenómeno particular, la reflexión individual y el trabajo en grupos pequeños con colaboración.

De esta manera la Web 2.0 funciona bien con el aprendizaje activo, ya que facilita la colaboración y el trabajo en grupo. Por otra parte, la naturaleza de las herramientas de texto, como los blogs y las wikis se prestan bien a la reflexión y, combinado con foros y hangouts, al debate.

Aprendizaje Social

Las teorías del aprendizaje social estudian el aprendizaje dentro de un contexto social. Las personas aprenden el uno del otro a través de una variedad de procesos tales como el aprendizaje por observación, la imitación y modelado. Los aspectos de interacción comunitaria de la Web 2.0 son atractivos para los teóricos del aprendizaje social, ya que permiten participar a los individuos en distintos niveles de la producción.

Aprendizaje constructivista

Estas teorías sostienen que los instructores, y el diseño de la instrucción, deben basarse en los conocimientos que los alumnos ya tienen.

Al igual que sucede con la teoría de la cultura participativa de Jenkins, los constructivistas sostienen que en el aprendizaje los estudiantes tiene posiciones como creadores activos. En este sentido las aplicaciones Web 2.0 pueden ser utilizadas para enfatizar el aprendizaje basado en problemas, la colaboración, la interactividad y el apoyo. Elementos todos que favorecen el protagonismo del estudiante.

\section{Los adoptadores tempranos de la web social en los procesos de gestión del aprendizaje en la formación online.}

La investigación formativa y basada en el diseño (Reigeluth y Frick, 1999) tiene entre otros el papel de orientar la acción en los ámbitos de gestión y organización, además de la práctica docente. Es pertinente pues incluir los resultados de una investigación llevada a cabo sobre modalidades de integración por entre los adoptadores tempranos (early adopters) como complemento a las conclusiones sobre los precedentes de integración 
de la web social y las consideraciones pedagógicas que hemos tratado en el apartado anterior. En lo que sigue resumiremos y comentaremos los resultados de la investigación llevada a cabo por el autor dentro del programa de Doctorado en Aplicaciones y Problemas Interdisciplinares de las Tecnologías de la Información y las Comunicaciones de La Escuela Politécnica Superior de la Universidad de Alcalá de Henares, y cuenta con el apoyo de la Red de Campus Virtuales Universitarios. Esta parte constituye un extracto de un artículo completo y detallado sobre el mismo tema (Zapata-Ros, 2014b). No reproduciremos aquí pues el contexto de la experiencia, ni otras características metodológicas. Simplemente se trata de reproducir un resultado parcial que es relevante con lo dicho en el apartado anterior y en el contexto del trabajo. Y que por otro lado justifique las conclusiones que a continuación expondremos.

En esta fase correlacionamos rasgos de perfil profesional y de perfil de uso de la web social, por los profesores, con valoración de las funciones pedagógicas y de diseño instruccional, a partir de las affordances que nos suministra, y hacemos estimaciones de las medias por intervalos de confianza, el estudio en su conjunto utilizará la metodología formativa.

La población y la muestra ha estado constituida por profesores universitarios en diversas funciones y tipos de docencia con unos rasgos comunes: Son profesores universitarios que utilizan LMS y los servicios de la web, en sus clases, integrados por una metodología docente con un diseño que contempla estos recursos en sus distintas dimensiones: Objetivos, actividades, evaluación, etc. De hecho son participantes de redes sociales profesionales, miembros de la Red Universitaria de Campus Virtuales o están vinculados a Campus Virtuales de universidades españolas, portuguesas e iberoamericanas. Se recogieron 154 cuestionarios válidos respondidos entre el $7 / 12 / 2012$ y el $18 / 07 / 2013$

En todos los casos que estudiamos correlacionamos variables construidas a partir del perfil del profesor con el uso que él o sus alumnos hacían de la web social, con variables sobre aceptación o uso integrado, diseño instruccional, etc. de la web social.

En un primer lugar correlacionamos la situación de los profesores con respecto al uso de web social por sus alumnos (clasificados en varias categorías progresivamente más selectivas: Tienen constancia... Está relacionado con ellos a través de esa red, etc.) con el factor " $5.3 \mathrm{El}$ uso del software social debe estar incluido en la organización instruccional (Guías didácticas, etc.) del programa formativo".

En este caso el Coeficiente de Correlación de Pearson ( $r$ ) fue 0,303833133

Es decir hay una correlación positiva entre las dos variables, existe una interdependencia pero es baja $(0,303833133$, recordemos que el Coeficiente de Correlación de Pearson está en un rango de 0 a 1). Lo cual se debe interpretar como que en alguna medida el que los profesores se impliquen en dar indicaciones a sus alumnos "sobre el uso y el alcance de uso en su asignatura" está correlacionado (es mayor que en el caso contrario) en que opinan que "El uso del software social debe estar incluido en la organización 
instruccional (Guías didácticas, etc.) del programa formativo". Pero el que sea relativamente bajo se debe interpretar como que hay algunos profesores de los que "dan indicaciones" que no son favorables a que el uso del software social debe estar incluido en la organización intruccional. $Y$ también de forma minoritaria, pero en mayor grado, hay profesores que no dan indicaciones a sus alumnos, suponemos que porque no hay oportunidad o porque no han llegado a ese grado de madurez en su innovación, pero que sí consideran que "El uso del software social debe estar incluido en la organización instruccional (Guías didácticas, etc.) del programa formativo".

Posteriormente repetimos el análisis de correlación del factor "El uso del software social debe estar incluido en la organización instruccional (Guías didácticas, etc.) del programa formativo" con las distintas situaciones de los profesores. Lo aplicamos y obtuvimos el siguiente cuadro

\begin{tabular}{|l|c|}
\hline & $\begin{array}{l}\text { Índice de correlación con 5.3 EI } \\
\text { uso del software social debe } \\
\text { estar incluido en la organización } \\
\text { instruccional (Guías didácticas, } \\
\text { etc.) del programa formativo }\end{array}$ \\
\hline $\begin{array}{l}\text { Tiene constancia de que sus alumnos utilizan redes } \\
\text { sociales de propósito general (Facebook, Twitter, } \\
\text { Google+,...) para ayudar a sus tareas o con fines de } \\
\text { conseguir un mejor resultado académico? }\end{array}$ & 0,245919402 \\
\hline Está relacionado con ellos a través de esa red & 0,205067166 \\
\hline $\begin{array}{l}\text { Obtienen indicaciones suyas sobre el uso y el alcance de } \\
\text { uso en su asignatura }\end{array}$ & 0,303833133 \\
\hline Evalúa su actividad en esa red & 0,23890356 \\
\hline $\begin{array}{l}\text { Coordina esa actividad con la que se sigue } \\
\text { habitualmente en el aula virtual (LMS o plataforma) }\end{array}$ & 0,301322411 \\
\hline
\end{tabular}

Los resultados en general suponen que entre este tipo de profesores (los innovadores tempranos) hay una alta consideración a que se debe producir la integración de la web social con repercusión e integración en el diseño instruccional y en la organización que se hace de forma institucional y que esta consideración de la integración, como algo posible y positivo para la eficiencia del aprendizaje, se producen en todos los casos, independientemente de cual sea el grado de implicación o de progreso de su implicación, si bien aumenta en la medida en que aumentan estos.

En la escala de situaciones de los profesores con respecto a sus alumnos

1. Tiene constancia de que sus alumnos utilizan redes sociales de propósito general (Facebook, Twitter, Google+,...) para ayudar a sus tareas o con fines de conseguir un mejor resultado académico?

2. Está relacionado con ellos a través de esa red

3. Obtienen indicaciones suyas sobre el uso y el alcance de uso en su asignatura

4. Evalúa su actividad en esa red 
5. Coordina esa actividad con la que se sigue habitualmente en el aula virtual (LMS o plataforma)

Existe una progresión no perfecta: Cada una de las situaciones implica la anterior excepto la cuarta. Se puede coordinar la actividad que siguen en la web social sin necesariamente de evaluar su actividad en esa red. Y supone una idea de engagement, de implicación y de confianza en las redes sociales como factor de mejora en el aprendizaje.

Si tenemos en cuenta esa progresión podemos concluir que en la medida que hay un mayor engagement la correlación con la idea de "incluir el software social en la organización instruccional (Guías didácticas, etc.) del programa formativo" aumenta

\begin{tabular}{|l|l|}
\hline $\begin{array}{l}\text { Tiene constancia de que sus alumnos utilizan redes } \\
\text { sociales de propósito general (Facebook, Twitter, } \\
\text { Google+,...) para ayudar a sus tareas o con fines de } \\
\text { conseguir un mejor resultado académico? }\end{array}$ & 0,245919402 \\
\hline Está relacionado con ellos a través de esa red & 0,205067166 \\
\hline $\begin{array}{l}\text { Obtienen indicaciones suyas sobre el uso y el alcance de } \\
\text { uso en su asignatura }\end{array}$ & 0,303833133 \\
\hline $\begin{array}{l}\text { Coordina esa actividad con la que se sigue } \\
\text { habitualmente en el aula virtual (LMS o plataforma) }\end{array}$ & 0,301322411 \\
\hline
\end{tabular}

En todo caso cuando el compromiso es mayor, como sucede en los casos en que "obtienen indicaciones" y "coordina esa actividad con la que se sigue habitualmente en el aula virtual", la correlación entre estos profesores y la idea favorable a incluir el software social en el diseño instruccional aumenta.

La correlación no obstante baja en la situación cuarta: "Evalúan la actividad de los alumnos en esa red". Ese resultado es consistente con un hecho posible: Los profesores que practican la evaluación formativa de aprendizajes a través de la web social, tiene cierta visión o sensación de fatiga, de incomodidad o de frustración, por no ver compensado suficientemente su trabajo. Una situación frecuente que coincide con la sensación de "quemado" que se produce en las vanguardias de los innovadores.

Y no es compatible con el hecho contrario: Que los profesores que además de coordinar y utilizar la web social para complementar la acción con el LMS, evalúen la actividad en la web social, sean más partidarios de incluirla en el diseño instruccional que los que no la evalúen no es compatible con las correlaciones obtenidas.

Esta conclusión nos abre un interesante elemento a investigar: ¿es compatible un diseño instruccional pleno, que incluya la evaluación de la producción en la web social, etc. realizado por profesores individuales con el marco actual, sin el apoyo de la institución, no solo con medios sino con una organización adecuada? Parece ser que este sería el punto de ruptura del voluntarismo, del engagement. 


\section{Conclusiones y recomendaciones}

Del análisis pormenorizado de lo visto se deducen conclusiones de diversa índole. Este trabajo, pues, nos ha situado ante informaciones y hechos suficientes para poder deducir la necesidad de en el futuro dejar abiertas las siguientes líneas de investigación y de profundización en aspectos de desarrollo, organización y de políticas universitarias que agrupamos en tres categorías: Contenidos, enseñanza y aprendizaje, gestión y tecnología.

\section{Contenidos}

Las instituciones deben considerar en su organización, en su gestión, en la financiación, etc. a largo plazo el procesamiento y el almacenamiento de forma adecuada para ser tratado de forma eficiente de los fondos documentales y de los materiales grises generados por los alumnos y profesores en la web social. Su capacidad de generar enseñanzas sobre buenas prácticas, resolución de problemas e investigaciones es algo que debe ser considerado a largo plazo. E incluso puede favorecer la práctica profesional de los alumnos después de abandonar la universidad.

En consecuencia las instituciones deberían considerar la organización, gestión, financiación, y mantenimiento de repositorios de estos materiales y de los derechos de autor (esto debiera comenzar por un estudio, pero las licencias Cretive Commons facilitan bastante las cosas). El fin es ver cómo se puede dar el servicio de acceso a este repositorio a usuarios potenciales en las materias de referencia. Debe contemplarse pues cómo se regulan los derechos de autor, citación, referencias, etc., aún en el caso de que sean de acceso abierto.

\section{Enseñanza y aprendizaje}

Las instituciones deberían considerar la organización, gestión, financiación, etc. de proyectos que investiguen la configuración de los repositorios generados en cuanto a metadatos, referencias que posibiliten el uso de los materiales en el diseño instruccional y en la organización curricular la explotación por materias, niveles, carreras, departamentos, centros de investigación educativa, etc. y para que sean también más accesibles para los estudiantes en procesos de aprendizaje a través del uso de las tecnologías sociales, incluyendo marcado y curación.

No consideramos prioritaria esta recomendación pero sí constatamos que hay un amplio sector que la cree necesaria: Se trata de impulsar (gestionar, organizar financiar etc.) la investigación de nuevas formas de enseñanza que utilizan sistemas de Web Social, 
teniendo en cuenta el desarrollo de nuevas herramientas de Web Social específicamente para el ámbito educativo, con facilidades que permiten la experimentación pedagógica.

Las instituciones deberían impulsar investigaciones, y crear un banco de datos sobre estudios de caso, que tenga como tema los impactos de la Web social en las formas de enseñar y de aprender. Esto debe incluir el impacto de la implementación de estas tecnologías en la estructura de las instituciones, el personal docente, personal de apoyo y en la configuración del trabajo de los estudiantes.

Las instituciones deben considerar con urgencia el apoyo a iniciativas que analizan en detalle la evaluación del trabajo en grupos que utilizan herramientas de la web social.

Las instituciones deben considerar el apoyo a iniciativas para desarrollar una gama de métodos de evaluación adecuado para su aplicación en el contexto del desarrollo del diseño instruccional utilizando la Web social. Esto podría ser en el contexto de un programa más amplio que abarque un modelo pedagógico completo, con métodos docentes, métodos de evaluación y herramientas sociales para el aprendizaje, enseñanza y la evaluación.

Todo esto concluiría en la recomendación de crear con carácter permanente de un departamento o servicio que impulsara y se responsabilizase de todas estas recomendaciones.

A esta recomendación le confiere un carácter relevante, al igual que justifica el crear una entidad propia, el vincular estas funciones y su naturaleza con la nueva configuración de una universidad tecnológica en la sociedad de la información que de soporte a las nuevas de docencia abierta on line, masiva o personalizada.

\section{Gestión}

Las instituciones deben considerar la implementación de estas iniciativas teniendo en cuenta los aspectos jurídicos de la propiedad intelectual, incluida la responsabilidad de las infracciones en materia de derechos de propiedad intelectual. $Y$ desarrollar guías de buenas prácticas para apoyar la creación abierta y reutilización de materiales.

Las instituciones deben contemplar la organización de un grupo de trabajo para examinar las formas de moderación (incluyendo moderación pares) y el control de contenido de la Web social. Y desarrollar guías de buenas prácticas para uso propio y para ofrecer a las instituciones consejos y ejemplos de buenas prácticas

Las instituciones deben contemplar los riesgos que suponen alojar a personas e instituciones asociadas en los servicios de la Web social, y las maneras en que los riesgos pueden ser controlados y mitigado. Esto podría hacerse en el contexto más amplio de los riesgos asociados al alojamiento en todos los servicios y en los equipos destinados a ellos.

Las instituciones deben tener medidas para prevenir el plagio, y para elaborar orientaciones sobre este mal en el uso de la Web social que apoya el aprendizaje, la docencia y la evaluación. 


\section{Tecnología}

Las instituciones deberían impulsar proyectos para el desarrollo de herramientas basadas en la Web para favorecer la evaluación formativa (el monitoreo continuo) de los procesos de grupo y del trabajo en grupo, teniendo en cuenta individual esfuerzo en el grupo. Y el desarrollo de herramientas de analítica de aprendizaje con carácter social.

\section{La falta de nuevos modelos pedagógicos y la creación de incertidumbre}

Ya hemos visto en el apartado dedicado a precedentes el consenso que hay sobre la incertidumbre. Es una percepción común de todos los académicos.

Actualmente las estructuras y procesos institucionales no están preparados para integrar las necesidades que se derivan de lo que se ha manifestado como necesario. Así sucede, por ejemplo, con la evaluación: hacen falta patrones y prácticas de evaluación que se centren por un lado en tareas que aporten juicios a la evaluación sumativa, desde una situación, la real, donde no se tienen en cuenta las prácticas pedagógicas de uso de la Web 2.0. Y por hace falta partir de una situación real: que los sistemas y las estrategias de evaluación están consagrados normativamente en España por pruebas y exámenes que no pueden dar soporte a procesos basados en Web Social.

Por último como justificación para el aprendizaje basado en la web social, y de paso un argumento sólido para persuadir a este sector de la industria que constituyen los LMS y al de la gobernanza académica, podemos utilizar lo que Keagan (2005a) Ilama "ley" de la educación a distancia según la cual

"No es con las tecnologías inherentes a las cualidades pedagógicas con las que se tienen éxito en la educación a distancia, sino con las tecnologías que están asumidas y son de uso generalizado por los ciudadanos".

Con solo cambiar "educación a distancia" por "educación superior en línea”

\section{Financing}

This research has not received any specific subsidies from funding agencies in the public, commercial or non-profit sectors.

\section{Financiación}

Esta investigación no ha recibido ninguna subvención específica de los organismos de financiación en los sectores públicos, comerciales o sin fines de lucro.

Conclusión del artículo: Septiembre de 2016

Presentación del artículo: 1 de octubre de 2017

Fecha de aprobación: 1 de de marzo de 2018

Fecha de publicación: 31 de marzo de 2018

Zapara-Ros, M. (2018). Gestión del aprendizaje y web social en la educación superior en línea. RED. Revista de Educación a Distancia, 57(7). Consultado el (dd/mm/aaaa) en http://www.um.es/ead/red/57/zapata.pdf 


\section{Referencias.-}

Alexander, B. (2008). Web 2.0 and Emergent Multiliteracies. Theory into Practice 47(2), 150-160.

Allen, I. E., \& Seaman, J. (2011). Going the distance: Online education in the United States, 2011. Sloan Consortium. PO Box 1238, Newburyport, MA 01950.

Anderson, T. (2006). Teaching a distance education course using educational social software. http://terrya.edublogs.org/2006/01/02/teaching-a-distance-educationcourse-using-educational-social-software/

Armstrong, J. and Franklin, T. (September 2008). A review of current and developing international practice in the use of social networking (Web 2.0) in higher education. Franklin Consulting. http://www.franklinconsulting.co.uk/LinkedDocuments/the\%20use\%20of\%20social\%20networking\%20i n\%20HE.pdf

Durán, S. P., Mezquita, J. P. G., \& García, T. J. (2013). Universitic 2013: situación actual de las TIC en el sistema universitario español: Diciembre 2013. Conferencia de Rectores de las Universidades Españolas, CRUE.

Franklin, T. and van Harmelen ,M. (2007, 28 May) Web 2.0 for Content for Learning and Teaching in Higher Education.

http://www.jisc.ac.uk/publications/reports/2007/web2andpolicyreport.aspx

Jenkins, H. (2009). Confronting the challenges of participatory culture: Media education for the 21st century. Mit Press.

Johnson, L., Adams Becker, S., Estrada, V., and Freeman, A. (2015). NMC Horizon Report: 2015 Higher Education Edition. Austin, Texas: The New Media Consortium. http://cdn.nmc.org/media/2015-nmc-horizon-report-HE-EN.pdf)

Keegan, D. (2005). The incorporation of mobile learning into mainstream education and training. http://mlearning.noekaleidoscope.org/public/mlearn2005/www.mlearn.org.za/CD/papers/keegan1.pdf

Kelly, B., 2008. Web 2.0 in Higher Education in the United Kingdom: Observations on the growth of Web 2.0 and social Web technologies. Franklin Consulting. Revisado en http://opus.bath.ac.uk/17702/ el 04/09/2013

Marwick, A. (September 2008) Web 2.0 in Higher Education in the United States of America. En A review of current and developing international practice in the use of social networking (Web 2.0) in higher education (p.91-122). Franklin Consulting. http://www.franklin- 
RED. Revista de Educación a Distancia, Núm. 57(7). 31-03-2018

DOI: http://dx.doi.org/10.6018/red/57/7

http://www.um.es/ead/red/57/zapata.pdf

consulting.co.uk/LinkedDocuments/the\%20use\%20of\%20social\%20networking\%20i

n\%2OHE.pdf

O'Reilly, T. (2006). Levels of the Game: The Hierarchy of Web 2.0 Applications, July 17. http://radar.oreilly.com/archives/2006/07/levels-of-the-game-the-hierarc.html or http://tinyurl.com/5vgrak

Ragins, B. R., \& Kram, K. E. (2007). The handbook of mentoring at work: Theory, research, and practice. Sage Publications.

Reigeluth, Ch. M. y Frick, T. W. (1999). Investigación formativa: una metodología para crear y mejorar teorías de diseño. En C. M. Reigeluth (Ed.) Diseño de la instrucción. Teorías y modelos. Un nuevo paradigma de la teoría de la instrucción (Parte II, 181100). Madrid: Aula XXI. Santillana.

Reigeluth, C. M., Watson, S. L., Watson, W. R., Dutta, P., Chen, Z., \& Powell, N. (2008). Roles for technology in the information-age paradigm of education: Learning management systems. Educational Technology, 48(6), 32-39

Reigeluth, C. (2012). Teoría instruccional y tecnología para el nuevo paradigma de la educación. RED, Revista de Educación a Distancia. Número 32. 30 de septiembre de 2012. Consultado el (dd/mm/aaa) en http://www.um.es/ead/red/32

Roblyer, M.D. et al (2010) Findings on Facebook in higher education: A comparison of college faculty and student uses and perceptions of social networking sites. The Internet and Higher Education.

http://www.sciencedirect.com/science/article/pii/S1096751610000278

Roblyer, M. D., \& Wiencke, W. (2003). Exploring the interaction equation: Validating a rubric to assess and encourage interaction in distance courses. The Journal of Asynchronous Learning Networks, 8(4), 24-37.

Rogers, E. M. (2010). Diffusion of innovations. Simon and Schuster. https://studieninteressierte.unihohenheim.de/uploads/tx_uniscripts/25720/A7020_KIM_2011.pdf\#page=37

Schwartz, H. (2009). Facebook: The new classroom commons? The Chronicle of Higher Education (pp. B13). October 2

The Stanford Persuasive Technology Lab (2007). The Stanford Facebook Class. http://credibilityserver.stanford.edu/captology/facebook/

Uceda, J., Mezquita, J. P. G., \& Largo, F. L. (2011). Universitic 2011: descripción, gestión y gobierno de las TI en el SUE: julio 2011. Conferencia de Rectores de las Universidades Españolas, CRUE.

Vázquez-Martínez, A. I., \& Cabero-Almenara, J. (2015). Las redes sociales aplicadas a la formación/Social networks applied to training. Revista complutense de educación, 26, 253. 
RED. Revista de Educación a Distancia, Núm. 57(7). 31-03-2018

DOI: http://dx.doi.org/10.6018/red/57/7

http://www.um.es/ead/red/57/zapata.pdf

Zapata, M. (2010). La investigación formativa y la investigación basada en el diseño: Dos perspectivas de alcance. RED - Revista de Educación a Distancia. Número 22.15 de mayo de 2010. Consultado el 29/08/13 en http://www.um.es/ead/red/22

Zapata-Ros, M. (2011a). Evaluación de la calidad en entornos sociales aprendizaje. RED. Revista de Educación a Distancia, (29) 1-10. Recuperado de http://www.redalyc.org/articulo.oa?id=54722116005 y http://www.um.es/ead/red/29/

Zapata-Ros, M. (2011b). La investigación y la edición científica en la web social: La ciencia compartida. Revista de Educación a Distancia-DUSC Número 3. Consultado el 26/08/2013 en http://www.um.es/ead/reddusc/3/zapata_e_ciencia.pdf

Zapata-Ros, M., 2012. Calidad y entornos ubicuos de aprendizaje. RED, Revista de Educación a Distancia. Número 31.- Revisado en http://www.um.es/ead/red/31/zapata_ros.pdf el 04/09/2013

Zapata-Ros, M. (2013a). Analítica de aprendizaje y personalización. Campus virtuales, 2(2), 88-118. http://www.uajournals.com/campusvirtuales/journal/3/7.pdf

Zapata-Ros, M. (2013b). “Evaluación de la calidad en entornos de aprendizaje de la web social y con tecnología ubicua". En ENTORNOS VIRTUALES Y APRENDIZAJE. Nuevas perspectivas de estudio e investigaciones. Págs. 313333. http://www.editorialeva.net/evya.html

Zapata-Ros, M. (2014a). Charles Reigeluth: la personalización del aprendizaje y el nuevo paradigma de la educación para la sociedad postindustrial del conocimiento. En Carreras, J. S., \& Albert, M. E. (Eds.). Pensadores de ayer para problemas de hoy: teóricos de las ciencias sociales (Vol. 6) (pp. 153-191). Nau Llibres.

Zapata-Ros, M. (2014b). Gestión del aprendizaje en Educación Superior y web social. RED, Revista de Educación a Distancia. Número 42. Número monográfico sobre "Experiencias y tendencias en affordances educativas de campus virtuales universitarios". 15 de septiembre de 2014. Consultado el (dd/mm/aaaa) en http://www.um.es/ead/red/42

Zapara-Ros, M. (2018). La universidad inteligente. RED. Revista de Educación a Distancia, 57(10). Consultado el (dd/mm/aaaa) en http://www.um.es/ead/red/57/zapata2.pdf 